\title{
Detection of Dermatophyte between Toes of Medical Students Wearing and not Wearing Socks in Universitas Padjadjaran using Direct Microscopic Examination
}

\author{
Venna Magarita, ${ }^{1}$ Ramlan Sadeli, ${ }^{2}$ Hendra Gunawan ${ }^{3}$ \\ ${ }^{1}$ Faculty of Medicine Universitas Padjadjaran ${ }^{2}$ Department of Microbiology Faculty of Medicine \\ Universitas Padjadjaran, ${ }^{3}$ Department of Dermatology and Venereology Faculty of Medicine \\ Universitas Padjadjaran/Dr. Hasan Sadikin General Hospital Bandung
}

\begin{abstract}
Background: Dermatophytes thrive in warm, humid, and moist areas. Wearing the same socks more than a day is one predisposing factor. The aim of this study is to detect the dermatophyte between the fourth and fifth toes of medical students of Universitas Padjadjaran, who wear and do not wear socks.

Methods: This was a cross-sectional descriptive study performed in Microbiology Laboratory of Faculty of Medicine Universitas Padjadjaran from September to October 2013. Direct microscopic examination using $10 \%$ Potassium Hydroxide (KOH) was performed on 50 medical students, 25 students who wear socks and 25 students who do not wear socks. The samples were taken from scraped skin between the fourth and fifth toes using the cellophane tape method. The sample size is obtained via stratified random sampling.

Results: From all participants, two students (4\%) showed positive result of dermatophyte hyphae and 1 student (2\%) showed positive result of dermatophyte hyphae and arthrospore. Of these three students, two students wear socks and one student does not wear socks. The two students who wear socks changed their socks more than a day.

Conclusions: Dermatophyte was detected in only a few medical students of Universitas Padjadjaran, who wear and do not wear socks. [AMJ.2015;2(4):480-4]
\end{abstract}

Keywords: Dermatophyte, direct microscopic examination, medical students

\section{Introduction}

Dermatophytosis is a superficial fungal infection involving stratum corneum of the skin, hair, or nails. ${ }^{1}$ Tinea pedis is one contagious dermatophyte infection of the toe webs and soles of the feet, mostly found between the fourth and fifth toes.,3 Dermatophytosis is common infection and occurs worldwide. It is estimated that over eight million people visit their primary health care physician for tinea related symptoms each year. ${ }^{4}$

Dermatophytes thrive on warm, humid, and moist areas. ${ }^{5}$ Some predisposing factors of getting infected include hot and humid weather, high perspiration, wearing occlusive footwear, and wearing the same socks for more than a day. Transmission of dermatophytes like Trichophyton rubrum, Trichophyton interdigitale and Epidemophyton floccosum is usually via feet. In this site, infections are often chronic and may remain subclinical for many years. ${ }^{4}$ Based on the observation, there are medical students of Universitas Padjadjaran who wear and do not wear socks. Few of them who wear socks do not replace it at least once a day, predisposing them to get dermatophyte infection. Therefore, the aim of this study is to detect the dermatophyte between the fourth and fifth toes of medical students of Universitas Padjadjaran who wear and do not wear socks using 10\% Potasium Hydroxide $(\mathrm{KOH})$ under direct microscopic examination.

\section{Methods}

This was a cross-sectional descriptive study conducted during September to October 2013 in Microbiology Laboratory, Faculty of Medicine, Universitas Padjadjaran.

With a stratified random sampling, a total of 50 male and female medical students

Correspondence: Venna Magarita, Faculty of Medicine, Universitas Padjadjaran, Jalan Raya Bandung-Sumedang Km.21, Jatinangor, Sumedang, Indonesia, Phone: +6287827590863 Email: veena_magarita@yahoo.com 
Table 1 Respondent Characteristic

\begin{tabular}{lcc}
\hline \multirow{2}{*}{ Characteristic } & Wear socks & Do not wear socks \\
\cline { 2 - 3 } Sex & $\mathbf{n = 2 5}$ & $\mathbf{n = 2 5}$ \\
$\quad$ Male & 15 & 0 \\
$\quad$ Female & 10 & 25 \\
Age (year old) & & \\
20 & 0 & 1 \\
21 & 13 & 10 \\
22 & 6 & 7 \\
23 & 5 & 5 \\
24 & 0 & 1 \\
25 & 0 & 0 \\
26 & 0 & 0 \\
27 & 1 & 1 \\
\hline
\end{tabular}

of Universitas Padjadjaran class of 2010 participated in this study; 25 students wearing socks, and the other 25 students not wearing socks. From this number, 15 of them were males and 35 females. The samples of students' informed consent were obtained for the study.

Samples were obtained using cellophane tape to collect the skin samples that had already been scraped between the fourth and fifth toes. The tape was then placed onto microscope slide that had a drop of $10 \% \mathrm{KOH}$. The slide was labeled into two; those who wore socks and those who did not wear socks, using a label pen.

The microscope slides were examined under a microscope (400x). Here, the hyphae (long, branching, rod-shaped filaments of uniformed width with separation lines) or arthrospore was visualized. Data was presented in a table in the form of frequency and percentage.

\section{Results}

All 25 students who wore socks used cotton socks. As for the students who did not wear socks, all 25 of them wore closed shoes.

The result of the direct microscopic examination using $10 \% \mathrm{KOH}$ showed positive findings in 3 out of the total 50 students (6\%). From 25 students wearing socks, 2 students were positive for dermatophyte and from 25 students who did not wear socks only 1 student was positive for dermatophyte (Table 3).

Out of all 50 students, 2 male students were
Table 2 Routine of Participants wearing Socks based on the Frequency of their changing Socks

\begin{tabular}{lc}
\hline Wearing socks & $\mathbf{n = 2 5}$ \\
\hline Changing socks & \\
everyday & 11 \\
more than a day & 14 \\
\hline
\end{tabular}

positive for dermatophyte (4\%). One male student showed positive for dermatophyte hyphae and 1 male student showed positive for dermatophyte hyphae and arthrospore. In females, only 1 participant out of 35 females

\section{Table 3 Percentage of Dermatophyte Presence}

\begin{tabular}{lc}
\hline Presence of Dermatophyte & $\mathbf{n = 3}$ \\
\hline Wear socks $(+)$ & 2 \\
Hyphae & 1 \\
Hyphae and arthrospore & 1 \\
arthrospore & 0 \\
Do not wear socks $(+)$ & 1 \\
Hyphae & 1 \\
Hyphae and arthrospore & 0 \\
arthrospore & 0 \\
\hline Note: Positive $(+):$ hyphae or arthrospore is found under \\
the microscope
\end{tabular}




\begin{tabular}{l}
$\begin{array}{c}\text { Table } 4 \text { Percentage of Routine of Changing } \\
\text { Socks in Students who were Positive } \\
\text { for Dermatophyte Hyphae or } \\
\text { Arthrospore }\end{array}$ \\
\hline $\begin{array}{c}\text { Presence of } \\
\text { dermatophyte in } \\
\text { those wearing socks }\end{array}$ \\
$\begin{array}{l}\text { Changing socks } \\
\text { everyday } \\
\text { more than a day }\end{array}$ \\
\hline
\end{tabular}

showed positive for dermatophyte hyphae. Of all 50 students, one female student was positive for dematophyte (2\%). Both students who wore socks and were positive for dermatophyte changed their socks more than a day (Table 4).

\section{Table 5 Percentage of Predisposing Factors Present in students who were Positive for Dermatophyte Hyphae or Arthrospore}

\begin{tabular}{lc}
\hline Predisposing Factors & $\mathbf{n = 3}$ \\
\hline Wear socks (+) & 2 \\
Male & 2 \\
Wear occlusive footwear & 2 \\
Damp feet & 1 \\
Have a pet at home (zoophilic) & 2 \\
Always in contact with soil (geophilic) & 0 \\
Live in hot and humid weather & 2 \\
Allergies & 0 \\
Immune compromise & 0 \\
Do not wear socks (+) & 1 \\
Male & 0 \\
Wear occlusive footwear & 1 \\
Damp feet & 1 \\
Have a pet at home (zoophilic) & 0 \\
Always in contact with soiln(geophilic) & 0 \\
Live in hot and humid weather & 1 \\
Allergies & 0 \\
Immune compromise & 0 \\
\hline thote : Positive (+): hyphae or arthrospore is found under \\
the
\end{tabular}

\section{Discussion}

Out of 50 students who participated in this study, only 3 students were positive for dermatophyte $(6 \%)$. This might be because the sensitivity for $\mathrm{KOH}$ is only $73.3 \%$ and the specificity is $77.7 \%$. Therefore, $\mathrm{KOH}$ is used only for complementary diagnosis of tinea pedis. The perfect gold standard for the diagnosis of the disease are positive $\mathrm{KOH}$ smear, positive culture, and also clinical exam showing tinea pedis. ${ }^{6}$ Furthermore, during microscopic view of $\mathrm{KOH}$ smear, textile fibers from socks may obfuscate proper discernment of dermatophyte. ${ }^{7}$ However, $\mathrm{KOH}$ smear is a good screening test for knowing the presence of the infection. ${ }^{6}$

In this study, hyphae itself was positive in 2 participants while arthrospore was not found in any participants under direct microscopic examination. Arthrospores are the asexual spores formed by the hyphae in the pathologic stage. ${ }^{8}$ Occasionally, hyphae are broken up into arthrospores that are first arranged as a string of pearls and later become detached. This phenomenon can be seen regularly in hair follicles, hair shafts, and nails, but less often in stratum corneum. In addition, hyphae and spores are transparent, and thus they are hard to detect. ${ }^{9}$

Based on this study, dermatophyte were positive in only one female and two males. The result of this study was similar to a study performed in 2012 discussing the comparison of dermatophytes and other agents of human dermatitis between males and females in Jos, Plateau State, Nigeria ${ }^{10}$. The study showed that $63.3 \%$ males had positive isolation for dermatophytes compared to $56.5 \%$ females. The reason underlying males to be more prone to dermatophyte infection than females is that male skin has larger pores, has more blood supply, and has more sebaceous glands that are active. Thus, males will be more prone to sweating causing more oily skin than females. Additionally, there is a difference between sex hormones, which make males more prone to be infected. Other than that, females are known to mount more forceful immune responses especially humor responses. In addition, the age of the onset of tinea pedis is in late childhood or young adult. ${ }^{4}$ In this study, all of the students were between the ages of 20 until 27 years old. Thus, it explains why in this age group positive result can be found.

Out of the three students who were positive for dermatophyte, two of the students wore 
socks (66.7\%) (Table 3). Those who did not wear socks were positive in one individual (33.3\%). Occlusion of the feet with socks can predispose one to a dermatophyte infection. ${ }^{11}$ Furthermore, socks are a way in which dermatophytes can spread. ${ }^{12}$ However, the type of socks can influence the amount of dermatophytes presence. Nylon socks shows larger number of dermatophyte isolated than the cotton and wool socks. Fibers of socks made from nylon and cotton are loose enough for dermatophytes to go through and thus, both are not good enough in preventing the adhesion of dermatophytes. ${ }^{13}$ However, in this study the type of socks worn by both students wearing socks and showing positive result for dermatophyte were the cotton socks. Socks worn should be replaced at least once a day. ${ }^{14}$ In this study, however, the students who wear socks and who were positive for dermatophyte changed their socks more than a day.

Dermatophytes grows predominantly in warm and humid areas. This is because warm and damp conditions help to promote the growth of dermatophytes. ${ }^{15}$ In this study, two students have damp feet, which consist of one student wearing socks and one student not wearing socks.

Tinea pedis is so contagious and is transmitted via direct or indirect contact with skin lesions of the infected people or contaminated environments. ${ }^{14,16}$ Based on their ecology, dermatophytes can be classified into anthropophilic. Dermatophytes spread from person to person either by direct contact or via fomites, zoophilic. Dermatophytes spread to humans from animals or via fomites and geophilic. Dermatophytes are found in the environment and spread when in contact with soil. ${ }^{4}$ Zoophilic organisms of dermatophyte are chiefly found in animals and cause marked inflammatory reactions in humans who have contact with infected cats, dogs, cattle, horses, birds or other animals. ${ }^{10}$ The students in this study spent most of their time in the faculty of medicine together and therefore are in close contact almost every weekday. However, most students here do not open and share their shoes and socks in the faculty and thus, lesser chance for a transmission event to occur. This explains why the result was only positive for dermatophyte in 3 out of 50 participants (6\%). Those three students might have contracted dermatophyte from anthropophilic, zoophilic or geophilic sources.

There are several limitations in this study. The detection of dermatophyte was done only using direct microscopic examination and not culture because culture of dermatophyte takes a very long time. ${ }^{17}$ Therefore, culture is not performed in this study. Other than that, the type of shoe material worn by the students is different. Wearing non-porous material can increase the moisture and temperature of the feet unlike wearing the open shoes. ${ }^{12,17}$ All the students in this study wore closed shoes, however the type of material worn is different. Thus, it might influence the presence of dermatophyte. Furthermore, some students might wear socks and might not wear socks alternately but they informed the researcher regarding the one most frequently worn. Some students might also forget if they were using any antifungal medication or having any underlying foot disease. Therefore, they were excluded in the study. In this study, it can be concluded that dermatophyte was detected in only a few medical students of Universitas Padjadjaran, who wore and did not wear socks. It is recommended that proper and hygiene behavior should be sought in order to reduce and eliminate the number of people infected with dermatophyte.

\section{References}

1. Newman WA. Dorlands pocket medical dictionary. 28th ed.Philadelphia: Elsevier Saunders; 2009. p. 232.

2. Tanaka K, Katoh T, Irimajiri J, Taniguchi H, Yokozeki H. Preventive effects of various types of footwear and cleaning methods on dermatophyte adhesion. J Dermatol. 2006;33(8):528-36.

3. Fauci A, Braunwald A, Kasper D, Hauser S, Longo D, Jameson J, Loscalzo J. Harrison`s principle of internal medicine. $17^{\text {th }}$ ed. New York: Mc Graw Hill Medical; 2008. p.1264

4. Wolff K, Johnson RA. Fitzpatricks color atlas and synopsis of clinical dermatology. 6th ed. New York: Mc Graw Hill Medical; 2009. p. 693, 4, 700, 1114.

5. PubMed Health. Athlete's foot (Tinea Pedis). 2013; [cited 2013 May 14] Available from: http://www.ncbi.nlm.nih. gov/pubmedhealth/PMH0001878/.

6. Levitt JO, Levitt BH, Akhavan A, Yanofsky H. The sensitivity and specificity of potassium hydroxide smear and fungal culture relative to clinical assessment in the evaluation of tinea pedis: a polled analysis. Dermatol Res Pract. 2010; 2010:764843.

7. Karan A, Alikhan A, Feldman SR. Microscopically differentiating dermatophytes from sock fibers. J Am Acad Dermatol. 2009; 61(6):1024-7. 
8. Cervantas O. Dermatophytosis. The center for Food Security and Public health,Iowa State University,Institude for International Cooperation in Animal Biologics, Iowa State University College of Veterinary Medicine; 2005.

9. Andersson EB. Dermatopathology. Berlin: Springer Verlag Berlin Heidelberg; 2006. p. 78.

10. Aleruchi C, Adesoji M, Louisa T, James D, Samson S, Emenike I.Comparison of dermatophytes and other agents of human dermatitis between males and females in Jos, Plateau State, Nigeria. Afr J Cln Exper Microbiol. 2012;13(2):84-9.

11. Gordon CC, Alimuddin Z. Manson`s tropical diseases. 22nd ed. Philadelphia: Elsevier Saunders. 2009.

12. Carlo CJ, MacWilliams BP. Tinea Pedis (Athlete's Foot); [cited 2013 May 13] Available from: http://www.bhchp.org/ BHCHP\%20Manual/pdf_files/Part1_PDF/
TineaPedis.pdf

13. Watanabe K, Taniguchi H, Nishioka K, Maruyama R, Katoh T. Preventive effects of various socks against adhesion of dermatophytes to healthy feet. Nihon Ishinkin Gakkai Zasshi. 2000;41(3):183-6.

14. Justad J. Athlete`s foot Best Practice Guideline; 2012; [Cited 2013 May 14] Available from: http://www. dphhs.mt.gov/dsd/ddp/documents / AthletesFoot.pdf.

15. Kumar V, Tilak R, Prakash P, Nigam C, Gupta R. Tinea pedis- an update. Asian J Medical Sciences. 2011; 2(2):134-8

16. Macit I, Ferdi T, Salih H, Turker G, Muhsin A. Epidemiology of tinea pedis and toenail tinea ungium in worshippers in the mosques in Adana, Turkey. J Dermatol. 2005;32(9):698-704.

17. Hainer BL. Dermatophyte infections. Am Fam Physician. 2003;67(1):101-109. 